\title{
Endogenous Brain-Derived Neurotrophic Factor Triggers Fast Calcium Transients at Synapses in Developing Dendrites
}

\author{
Susanne B. Lang, Valentin Stein, Tobias Bonhoeffer, and Christian Lohmann \\ Max Planck Institute of Neurobiology, 82152 Martinsried-München, Germany
}

\begin{abstract}
Brain-derived neurotrophic factor (BDNF) is involved in many aspects of the formation of functional neuronal networks. BDNF signaling regulates neuronal development not only globally, at the level of entire neurons or networks, but also at a subcellular level and with high temporal specificity; however, the spatiotemporal characteristics of intrinsic BDNF signaling are essentially unknown. Here, we used calcium imaging to directly observe intrinsic BDNF signaling in developing hippocampal neurons. We found that blocking intrinsic BDNF signaling with function-blocking BDNF antibodies $(\alpha \mathrm{BDNF})$ or K252-a reduced the frequency of spontaneously occurring fast and localized calcium rises in dendrites. Conversely, focal application of BDNF evoked fast and local dendritic calcium transients, which required activation of TrkB (tropomyosin-related kinase B) receptors as well as activation of voltage-gated sodium and calcium channels. Virus-mediated expression of PSD-95:CFP (postsynaptic density-95 tagged with cyan fluorescent protein) revealed that spontaneous local calcium transients occurred frequently at postsynaptic sites along the dendrite. The frequency of synaptically localized calcium transients was specifically reduced by blocking intrinsic BDNF signaling, whereas nonsynaptic calcium rises were not affected. Furthermore, focal BDNF delivery evoked localized and fast calcium elevations specifically at postsynaptic sites. Together, our results demonstrate that BDNF-dependent calcium signaling in developing hippocampal neurons is fast and occurs at synapses. These temporal and spatial characteristics of intrinsic BDNF signaling as well as its relative abundance renders BDNF an ideal signaling molecule in the establishment of specific synaptic connectivity and functional neuronal networks.
\end{abstract}

Key words: calcium signaling; development; BDNF; TrkB; postsynaptic site; hippocampus

\section{Introduction}

In the CNS, neurotrophins are well known for their ability to support neuronal survival and to control differentiation during development (Bibel and Barde, 2000; Huang and Reichardt, 2001). Besides these classical long-lasting effects, recent evidence has revealed acute effects of neurotrophins on neuronal plasticity. In particular, brain-derived neurotrophic factor (BDNF) and its receptor tropomyosin-related kinase $\mathrm{B}(\mathrm{TrkB})$ affect neuronal morphology in cortical circuitries through the control of dendritic and axonal branching in an activity-dependent manner (McAllister et al., 1995; Gorski et al., 2003) (for review see, McAllister et al., 1999). Moreover, BDNF signaling can modulate synaptic strength: it exerts acute effects on synaptic transmission (Lohof et al., 1993; Kang and Schuman, 1995; Levine et al., 1995, 1998) (for review, see Poo, 2001) and is involved in the induction and maintenance of hippocampal long-term potentiation (LTP) (Korte et al., 1995, 1996; Patterson et al., 1996; Kossel et al., 2001; Kovalchuk et al., 2002), as well as in the stabilization of synapses (Hu et al., 2005).

Received Aug. 18, 2006; revised Dec. 8, 2006; accepted Dec. 19, 2006.

We thank Ann Marie Craig (Vancouver, British Columbia, Canada) for the generous gift of the PSD-95:CFP and Hans Thoenen and Mark Hübener for comments and critical reading of this manuscript. We thank Nicole Stöhr for preparation and maintenance of slice cultures and Thomas Kleindienst for developing a Matlab program used in our data analysis. We also thank Sydney Cambridge for providing the BDNF antibodies.

Correspondence should be addressed to Christian Lohmann, Max Planck Institute of Neurobiology, Am Klopferspitz 18, 82152 Martinsried-München, Germany. E-mail: Iohmann@neuro.mpg.de. DOI:10.1523/JNEUROSCI.3590-06.2007

Copyright $\odot 2007$ Society for Neuroscience $\quad$ 0270-6474/07/271097-09\$15.00/0
BDNF influences synaptic plasticity not only at a cellular but also at a subcellular level. For instance, apical and basal dendrites of the same pyramidal cells respond differently to the same neurotrophin (McAllister et al., 1995). Furthermore, BDNF release from a single cell has been shown to directly induce local dendritic growth and branching in nearby neurons in a distancedependent manner within a relatively short time (Horch and Katz, 2002). In addition, it has been reported that local application of BDNF and simultaneous presynaptic activation mediate LTP in mature dentate granule cells (Kovalchuk et al., 2002). The expression and secretion of BDNF is known to be regulated by neuronal activity (Balkowiec and Katz, 2002; Gartner and Staiger, 2002), and BDNF release occurs at synapses, possibly at presynaptic or postsynaptic compartments or both (Hartmann et al., 2001; Kohara et al., 2001; Gartner et al., 2006) (for review, see Lessmann et al., 2003). These findings suggest that BDNF signaling is precisely regulated in space and time.

Calcium as an important second messenger acts downstream of BDNF and is thought to mediate some of its effects in a specific, fast and localized manner. For example, it has been shown that intracellular calcium signaling conveys the potentiating effect of BDNF at hippocampal synapses (Kang and Schuman, 2000; Kovalchuk et al., 2002). Moreover, the application of exogenous BDNF is known to induce a transient elevation in the intracellular calcium concentration, predominantly as a result of calcium release from internal stores (Berninger et al., 1993) but also dependent on calcium influx from the extracellular space (Marsh and Palfrey, 1996). 
Although there is a substantial amount of knowledge about the cellular responses to exogenous BDNF, it has not yet been studied how endogenous BDNF signaling affects neuronal calcium responses. Therefore, we set out to observe directly intrinsic BDNF-induced calcium signaling in developing pyramidal neurons of hippocampal slice cultures. We asked whether spontaneous local calcium events are triggered by BDNF and investigated the mechanisms underlying BDNF-mediated calcium signaling. We found that intrinsic BDNF signaling elicited rapid and spatially restricted calcium rises in dendrites. BDNF-dependent local calcium transients required activation of TrkB receptors as well as voltage-sensitive sodium and calcium channels. Moreover, we observed that most of the BDNF-dependent spontaneous local calcium transients occurred at postsynaptic sites along the dendrite. Accordingly, focal BDNF delivery evoked localized calcium elevations specifically at single postsynaptic sites. Together, our data show that BDNF triggers fast and localized calcium transients in developing neurons, which preferentially occur at synapses.

\section{Materials and Methods}

Preparation of hippocampal slice culture. Hippocampal organotypic cultures from postnatal day $0(\mathrm{P} 0)$ to $\mathrm{P} 2$ rats were prepared following the method of Stoppini et al. (1991). After decapitation, the hippocampi were dissected in ice-cold Gey's balanced salt solution, containing $0.5 \mathrm{ml}$ of kynurenic acid and $0.5 \mathrm{ml}$ of glucose, $\mathrm{pH}$ 7.2. Transversal slices were cut $(400 \mu \mathrm{m})$ using a tissue chopper (McIlwain). Slices were plated onto Millicell membrane inserts (CM; Millipore, Billerica, MA) and incubated with DMEM medium containing $25 \%$ horse serum at $37^{\circ} \mathrm{C}, 7 \% \mathrm{CO}_{2}$ for up to $3 \mathrm{~d}$.

Virus-mediated gene transfer. Postsynaptic density-95 tagged with cyan fluorescent protein (PSD-95:CFP) was expressed using viral infection. PSD-95:CFP (Graf et al., 2004) was cloned into the pSCA vector (DiCiommo and Bremner, 1998). Semliki forest viral particles were produced by transfecting pSCA-PSD-95:CFP and pHelper in a 1:2 ratio into human embryonic kidney HEK 293 cells. At $48 \mathrm{~h}$ after transfection, the supernatant was harvested, aliquoted, and stored at $-80^{\circ} \mathrm{C}$. The virus was activated by $\alpha$-chymotrypsin treatment for $45 \mathrm{~min}$ and injected into the $\mathrm{CA} 3$ region.

Single-cell electroporation of CA3 pyramidal cells and bolus loading of the $C A 3$ region. The recording chamber was temperature controlled at $35^{\circ} \mathrm{C}$ and perfused with HBSS consisting of the following (in $\mathrm{mM}$ ): $14.61 \mathrm{CaCl}_{2}, 4.93$ $\mathrm{MgCl}_{2}, 4.07 \mathrm{MgSO}_{4}, 53.33 \mathrm{KCl}, 4.41 \mathrm{KH}_{2} \mathrm{PO}_{4}, 4.2 \mathrm{NaHCO}_{3}, 1379.31 \mathrm{NaCl}$, $3.36 \mathrm{Na}_{2} \mathrm{HPO}_{4}$, and $55.56 \mathrm{D}$-glucose. The high-affinity calcium indicator Oregon Green BAPTA-1 (250 $\mu \mathrm{M}$, dissolved in distilled water, hexapotassium salt; Invitrogen, Carlsbad, CA) was electroporated into single neurons according to the method for dye electroporation (Haas et al., 2001; Rathenberg et al., 2003; Lang et al., 2006). Patch pipettes with a tip diameter of $\sim 1$ $\mu \mathrm{m}$ and square voltage pulses ( $10 \mathrm{~V}, 20 \mathrm{~ms}$ duration) were used to fill individual neurons. For bolus loading (Stosiek et al., 2003), $50 \mu \mathrm{g}$ (one vial) of Oregon Green 488 BAPTA-1 AM (Invitrogen) was dissolved in $5 \mu \mathrm{l}$ of DMSO containing $10 \%$ of pluronic acid. After sonicating for $1 \mathrm{~min}, 50 \mu \mathrm{l}$ of culture medium was added, and the solution was again sonicated. The tip of a patch pipette was filled with the dye solution, and the CA3 region of hippocampal slices on culture inserts was loaded by positioning the pipette tip in the stratum radiatum/stratum lacunosum-moleculare and applying short pressure pulses with a Picospritzer (General Valve, Fairfield, NJ). After a $1 \mathrm{~h}$ incubation, slices were used for experiments.

Imaging. Recordings were acquired using an Axioplan-2 microscope (Zeiss, Oberkochen, Germany) and a cooled CCD camera (VisiCam QE; Visitron Systems, Puchheim, Germany) controlled with MetaMorph software (Universal Imaging Corporation, West Chester, PA). Images were obtained at $0.3-10 \mathrm{~Hz}$ with either a Zeiss $0.95 / 63 \times$ or an Olympus Optical (Tokyo, Japan) $0.80 / 40 \times$ water immersion objective provided by H. Thoenen (Max Planck Institute of Neurobiology, Martinsried-München, Germany).

Application of BDNF and pharmacological agents. BDNF (26 $\mu \mathrm{l}, 15$ $\mu \mathrm{g} / \mathrm{ml}$ in PBS, containing $0.1 \%$ BSA to prevent binding to the storage and application ware; provided by $\mathrm{H}$. Thoenen) was applied using a $100 \mu \mathrm{l}$ pipette (final concentration of $200 \mathrm{ng} / \mathrm{ml}$ ). Superfusion was interrupted during BDNF bath application; control experiments showed that interruption of superfusion alone did not affect calcium activity for the recording period (data not shown). Alternatively, BDNF was applied focally to the apical dendrites with pressure pulses through a micropipette using a Picospritzer (pulse duration of $40 \mathrm{~ms}$ ). The BDNF application pipette $(\sim 1 \mu \mathrm{m}$ tip diameter) was placed at a distance of $\sim 10-20 \mu \mathrm{m}$ from the dendrite, and three pulses were applied within $1 \mathrm{~min}$. In control experiments, either PBS with $0.1 \%$ BSA or heat-inactivated BDNF (30 min at $95^{\circ} \mathrm{C}$ ) was pressure applied to the dendrite.

The protein kinase antagonist K-252a (200 nm; Alexis Biochemicals, San Diego, CA) and the function-blocking mouse monoclonal antibody against BDNF ( $\alpha$ BDNF) were bath applied $(4 \mu \mathrm{g} / \mathrm{ml}$, clone number 9 , IgG) (Kolbeck et al., 1999; Kossel et al., 2001). For $\alpha$-BDNF experiments, tubing was siliconized for $1 \mathrm{~h}$, dried, and extensively washed to prevent adhesion of the antibody to tubing surfaces. Additionally, BSA $(0.5 \mathrm{mg} /$ $\mathrm{ml}$ ) was added to the antibody solution. All other pharmacological agents were diluted in HBSS and bath applied: $1 \mu \mathrm{M}$ tetrodotoxin (TTX), $5 \mu \mathrm{M}$ cadmium chloride $\left(\mathrm{CdCl}_{2}\right), 20 \mu \mathrm{M}$ cyclopiazonic acid (CPA), and $3 \mu \mathrm{M}$ SKF 96365 hydrochloride [1-2-(4-methoxyphenyl)-2-[3-(4-methoxyphenyl)propoxy] ethyl- $1 H$-imidazole]. Tetanus toxin (TeTX) at $20 \mathrm{~nm}$ was added to the culture medium at least $12 \mathrm{~h}$ before the recordings.

Image analysis. Changes in $\left[\mathrm{Ca}^{2+}\right]_{\mathrm{i}}$ are derived from $\Delta F / F_{0}$, where $F_{0}$ is the baseline fluorescence, which is typically taken from the first 20 images of a recording. Global calcium transients were defined as fluorescence increases of at least $10 \%$ observed in all structures of the cell within the same focal plane. Locally occurring calcium transients were defined as local rises in fluorescence of $>5 \%$ with a spatial extent of $1-30 \mu \mathrm{m}$ along the dendrite. For analysis of the BDNF application experiments, changes caused by dendrite shifts attributable to the pressure pulse application were discarded. For statistical analysis, Wilcoxon's matched-pairs test was used.

Western blot. Hippocampal tissue from P3 rats was homogenized in gradient buffer (containing $0.32 \mathrm{M}$ saccharose and $1 \mathrm{mM} \mathrm{MgCl}_{2}, \mathrm{pH} 7.4$ ), $2 \times$ sample buffer $(0.125 \mathrm{~m}$ Tris $\mathrm{pH} 6.8,2 \%$ SDS, $20 \%$ glycerol, and $0.001 \%$ bromphenolblue), DDT, and a protease inhibitor cocktail (Sigma, Deisenhofen, Germany). HEK 293 cells served as controls. Aliquots of the lysates were then subjected to SDS-PAGE (6\% acrylamide) and transferred to nitrocellulose membranes (transfer membranes; Millipore). The blots were incubated in blocking buffer of $0.05 \%$ Tween 20 and $0.5 \%$ gelatin in $10 \times$ Tris-buffered saline at $4^{\circ} \mathrm{C}$ overnight. After blocking, the nitrocellulose membrane was incubated with either a rabbit anti-TrkB antibody (1:50; Santa Cruz Biotechnology, Santa Cruz, CA) or a rabbit anti-actin (1:100; Sigma) for $1 \mathrm{~h}$ at room temperature. After incubation with the secondary antibody $(1 \mathrm{~h}$, room temperature, $1: 10,000$, peroxidase labeled anti-rabbit antibody), binding of the antibody was detected using an ECL detection kit (Amersham Biosciences, Braunschweig, Germany).

Immunohistochemistry. After viral infection with PSD-95:CFP, slices were fixed at $4^{\circ} \mathrm{C}$ overnight in paraformaldehyde ( $4 \%$ in 0.1 sodium phosphate buffer). After washing for $3 \mathrm{~h}$ in phosphate buffer (PB), slices were preincubated in a blocking solution (4\% Triton X-100, 1.5\% horse serum, and $0.1 \% \mathrm{BSA}$ in $\mathrm{PB}, 4^{\circ} \mathrm{C}$, overnight) and then incubated in the primary antibody [rabbit anti-synapsin (Chemicon, Temecula, CA), diluted 1:500 in 4\% Triton X-100, 1.5\% horse serum, and 0.1\% BSA in PB] for 7-10 d. Slices were rinsed ( $3 \mathrm{~h}$ in $\mathrm{PB}$ ) and then incubated for 2-3 d with the secondary antibody (anti-rabbit FITC, 1:50 in PB, $4^{\circ} \mathrm{C}$ ). Stacks of high-resolution images (voxel size, $x-y-z, 0.18 \times 0.18 \times 0.25 \mu \mathrm{m}$ ) from PSD-95:CFP-infected dendrites and anti-synapsin-labeled structures were obtained with a confocal microscope [Leica (Nussloch, Germany) SP2, sequential acquisition mode, $1.25 / 40 \times$ oil immersion objective]. Quantification of colocalization was performed using NIH ImageJ software. Puncta were considered colocalized when pixels of PSD-95: CFP puncta ("red" channel) and synapsin puncta ("green" channel) overlapped in high-magnification $x-y$ and orthogonal $(y-z)$ views ("yellow" pixels). The specificity of colocalization was tested using the reverse order of the synapsin stack rotated by $180^{\circ}$. 

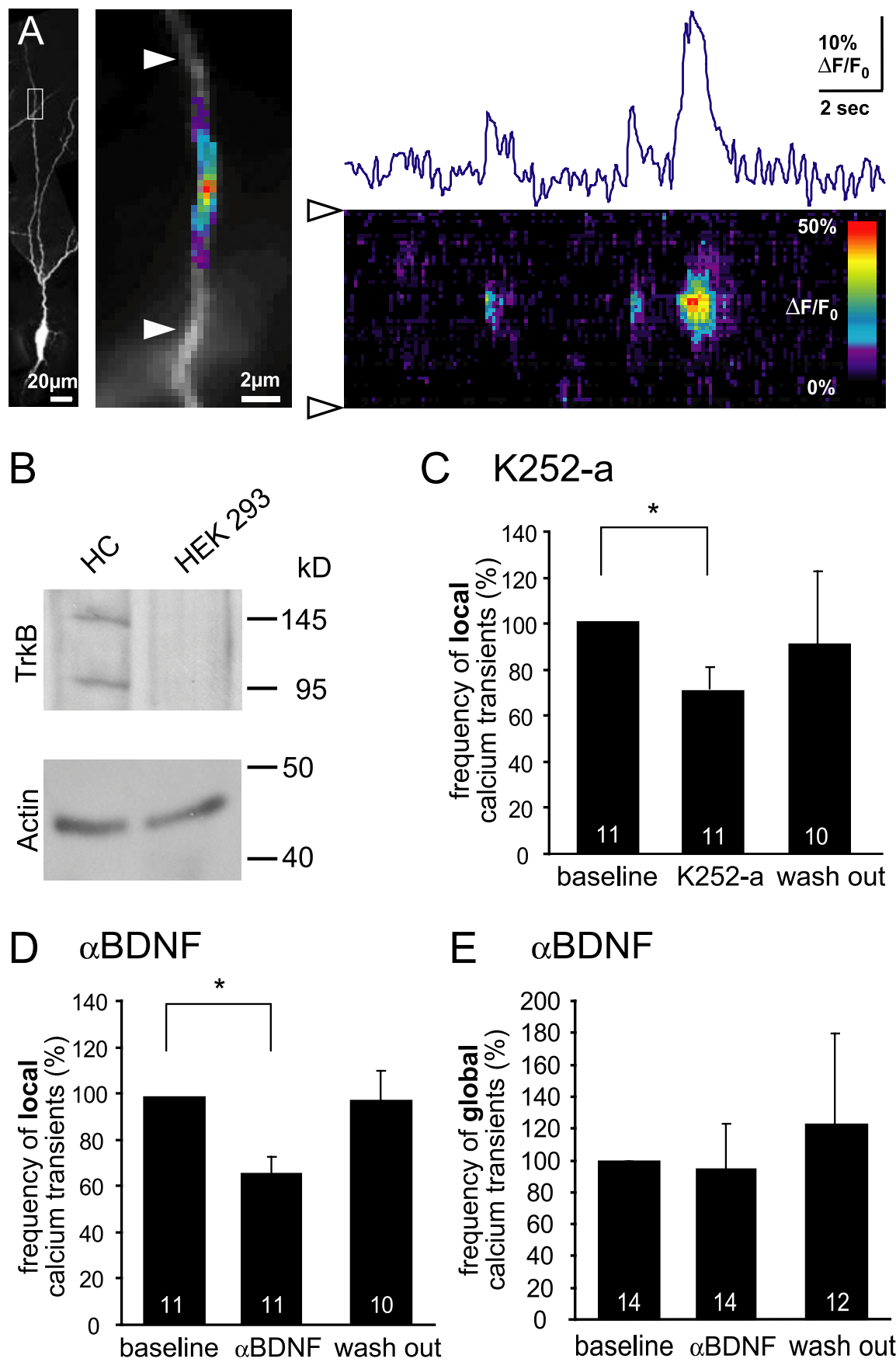

Figure 1. Frequency of spontaneously occurring local calcium transients in dendrites of developing hippocampal neurons is decreased by BDNF antibody or K252-a application. $\boldsymbol{A}$, Electroporated single CA3 pyramidal neuron (P2 + 3 din vitro) filled with Oregon Green BAPTA-1. Enlarged part of the dendrite shows spontaneous local increase in $\left[\mathrm{Ca}^{2+}\right]_{\mathrm{i}}$, represented as $\Delta F / F_{0}$ in pseudocolor. Arrowheads indicate the extent of the measured dendritic segment used for pseudo line scan and trace. $\boldsymbol{B}$, Western blot analysis of TrkB receptor and actin in hippocampal lysates from developing rats (P2). Both full-length TrkB and truncated TrkB (molecular weights of 145 and $95 \mathrm{kDa}$, respectively) were present in the hippocampus (HC) but absent in the control (lysate of HEK 293 cells). Actin served as the loading control. C, Frequency of spontaneous local calcium transients before, during, and after bath application of K252-a normalized to baseline frequency. Calcium activity is significantly reduced during K252-a application. $n=$ 11 neurons; error bars indicate SEM $\left({ }^{*} p<0.05\right)$. $D$, Similarly, bath application of $\alpha$ BDNF causes a significant and reversible decrease in the frequency of spontaneous local calcium transients. $n=11$ neurons $\left({ }^{*} p<0.05\right)$. $\boldsymbol{E}$, The frequency of spontaneous global calcium transients is unchanged during BDNF antibody application.

\section{Results}

Endogenous BDNF induces spontaneous local calcium transients

In our study, we focused on the first postnatal week when dendrites and axons undergo dynamic structural remodeling and form new synapses in vivo and in organotypic slice cultures (Dailey and Smith, 1996; Fiala et al., 1998). We observed spontaneous local rises of $\left[\mathrm{Ca}^{2+}\right]_{i}$ in CA3 pyramidal neurons, which were labeled by single-cell electroporation (Fig. 1A). These local calcium transients occurred at a mean frequency of $73.8 \pm 10.8$ per minute and millimeter of dendrite. The average duration of such calcium rises was $680 \pm 120 \mathrm{~ms}$, and they were restricted to small stretches of $9.0 \pm 0.6 \mu \mathrm{m}$ of the dendrite. The amplitude of the transients (measured as $\Delta F / F_{0}$ ) was $17.3 \pm 0.6 \%$, and their rise time was zero to four frames of $100 \mathrm{~ms}$ (median of two frames). Besides local calcium transients, hippocampal neurons also generated global calcium transients occurring in the soma, dendrite, and axon, presumably representing network activity (Garaschuk et al., 1998).

We investigated the role of endogenous BDNF for spontaneous local calcium signaling by interfering with the BDNFTrkB signaling pathway. After confirming the presence of TrkB, the principal receptor for BDNF, in the developing hippocampus by Western blot analysis (Fig. $1 B$ ), we used either K252-a, which blocks tyrosine phosphorylation of Trk receptors, including TrkB, or functionblocking BDNF antibodies to inactivate endogenous BDNF. During bath application of 200 nM K252-a, we observed a significant and reversible reduction in the frequency of spontaneous local calcium transients $(-26.2 \pm 9.4 \% ; n=11 ; p<$ 0.05) (Fig. 1C). Temporal and spatial characteristics of local calcium transients were not changed. The frequency of global calcium transients remained stable. Similarly, bath application of BDNF antibodies (4 $\mu \mathrm{g} / \mathrm{ml}$ ) diminished the frequency of spontaneously occurring local calcium transients significantly $(-33.8 \pm 7.4 \%$; $n=11 ; p<0.05$ ) (Fig. $1 D)$. In contrast, the frequency of the global signals did not change significantly $(-5.4 \pm 28.5 \%$ ) (Fig. $1 E)$. These results show that interfering with the BDNF-TrkB signaling pathway reduces the frequency of spontaneous local calcium signaling, indicating that endogenous BDNF generates intracellular calcium signals via $\operatorname{TrkB}$ receptor activation.

Exogenous BDNF strongly increases the frequency of calcium transients

The observation of a reduced frequency of local calcium transients during inhibition of the BDNF-TrkB signaling pathway led us to ask whether additional exogenous BDNF would enhance calcium activity. We therefore applied exogenous BDNF (final concentration, $200 \mathrm{ng} / \mathrm{ml}$ ) into the bath solution of the recording 
chamber and observed a strong and reversible increase in the frequency of global calcium transients $(+239.4 \pm 88.9 \% ; n=$ $8 ; p<0.05)$ (Fig. $2 A, B)$. In contrast to global calcium rises, the frequency of local calcium transients did not increase significantly during exogenous BDNF application $(+41.9 \pm 19.9 \% ; n=12$ ) (Fig. $2 C$ ).

The increase in global activity might be a direct cellular response to BDNF. Alternatively, BDNF may modulate the network activity of the hippocampal circuit, resulting in an increase in global calcium activity (Sakai et al., 1997). To test whether the strong increase in the frequency of global calcium transients was a direct effect or caused by enhanced network activity, we used bolus loading (Stosiek et al., 2003) of Oregon Green BAPTA-1 AM to visualize network activity in CA3 pyramidal neurons. After fast BDNF application, we observed a sustained increase in the frequency of calcium transients in many neurons (before, $0.8 \pm 0.3$; after, $4.9 \pm 0.8$ global transients/min; $p<0.05 ; n=8$ slices; 80 cells) (Fig. 2D,F). The calcium transients were highly correlated across neurons, indicating that they reflected an increase in network activity. Averaging the responses from the entire set of cells showed that there was also an immediate but transient calcium response on BDNF application (Fig. $2 G$ ). When the experiments were repeated in slices preincubated $(12 \mathrm{~h})$ in TeTX to block network activity, BDNF

Figure 2. BDNF bath application causes a strong increase in the frequency of global calcium transients. $A$, Spontaneous $\left[\mathrm{Ca}^{2+}\right]_{i}$ changes in the dendritic segment marked with the arrowheads are shown in pseudo line scans as $\Delta F / F_{0}$. Line scans are shown before and during BDNF bath application (at 3 different time points) and after washout. $\boldsymbol{B}$, The frequency of spontaneously occurring global calcium transients is significantly $\left({ }^{*} p<0.05\right)$ increased during bath application of BDNF ( $n=8$ neurons, values normalized to baseline frequency). $C$, Frequency of spontaneous local calcium transients is not significantly increased during BDNF bath application $(n=12$ neurons). $\boldsymbol{D}$, Bolus loading of (A3 neurons in control slices shows an acute increase in $\left[\mathrm{Ca}^{2+}\right]_{i}$ during BDNF bath application and a delayed increase in correlated network activity (traces correspond to the encircled neurons in the left image). $\boldsymbol{E}$, Neurons in slices incubated for $12 \mathrm{~h}$ in TeTX also reveal an immediate increase in $\left[\mathrm{Ca}^{2+}\right]_{\mathrm{i}}$ to BDNF bath application but otherwise remain silenced. $\boldsymbol{F}$, Frequency of global calcium transients in control- and TeTX-incubated slices before and after BDNF bath application (80 neurons from 8 slices; control slices: before, $0.8 \pm 0.3$; after, $4.9 \pm 0.8$ global calcium transients per minute; TeTX-incubated slices: before, $0.07 \pm$ 0.02 ; after, $0.17 \pm 0.06$ global calcium transients minute; $\left.{ }^{*} p<0.05\right)$. G, Averaged acute calcium responses to fast BDNF bath application in control slices and in TeTX-incubated slices (80 neurons from 8 slices).
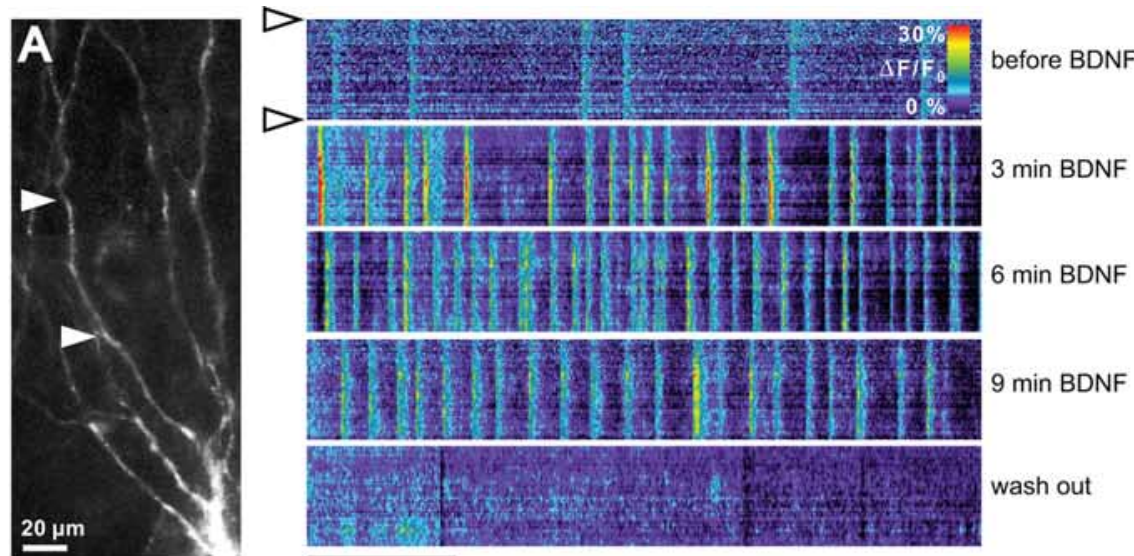

wash out
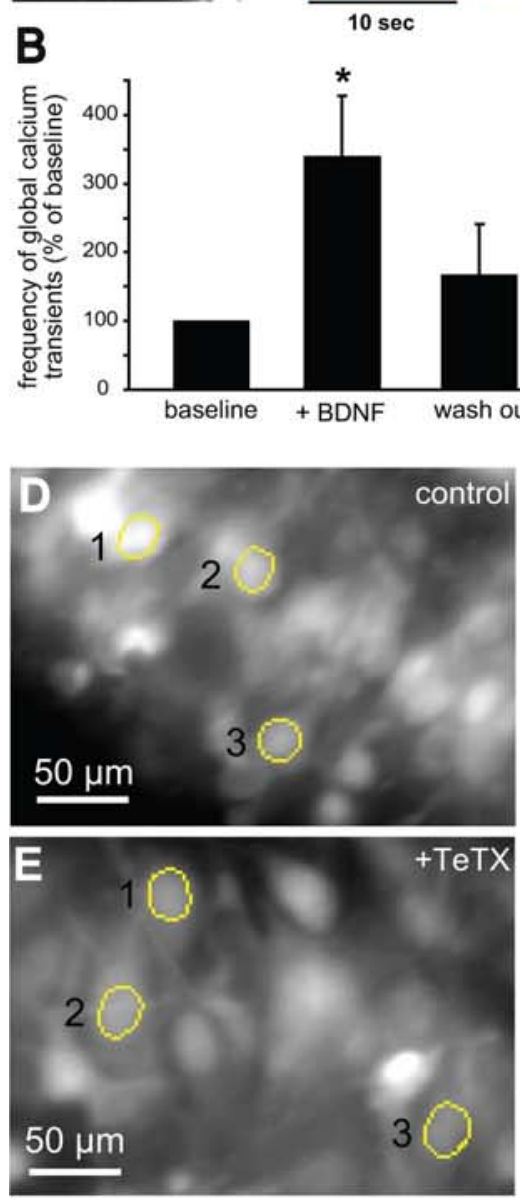

$\mathbf{F}$

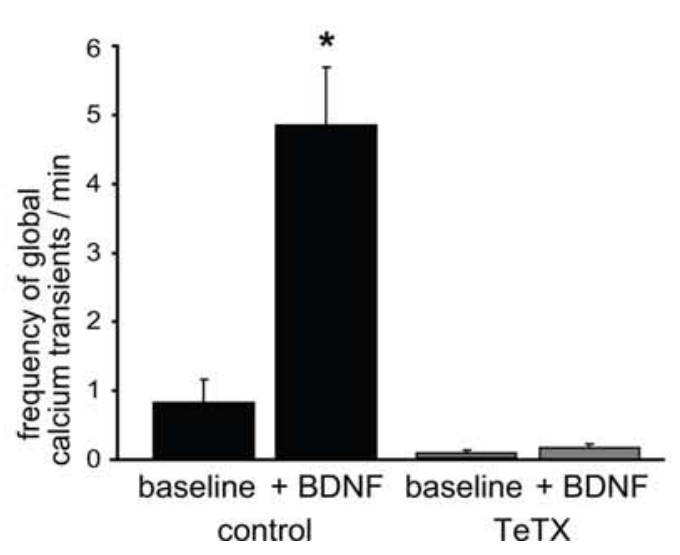

\section{C}
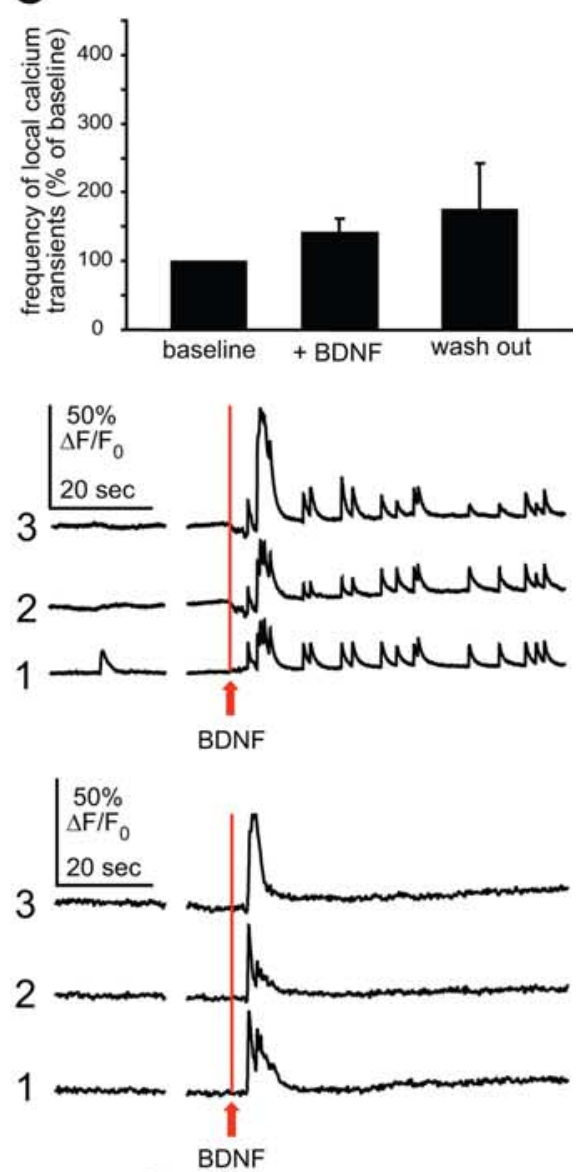

G

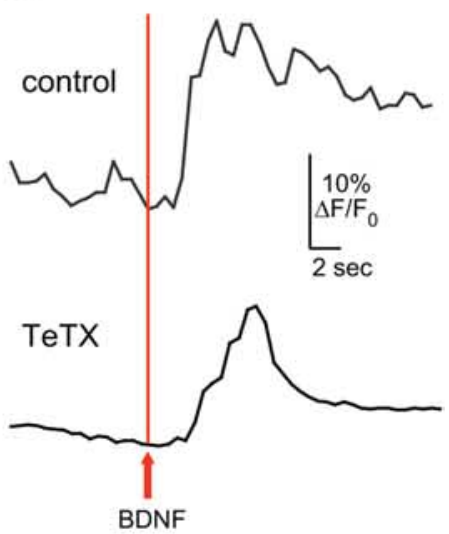



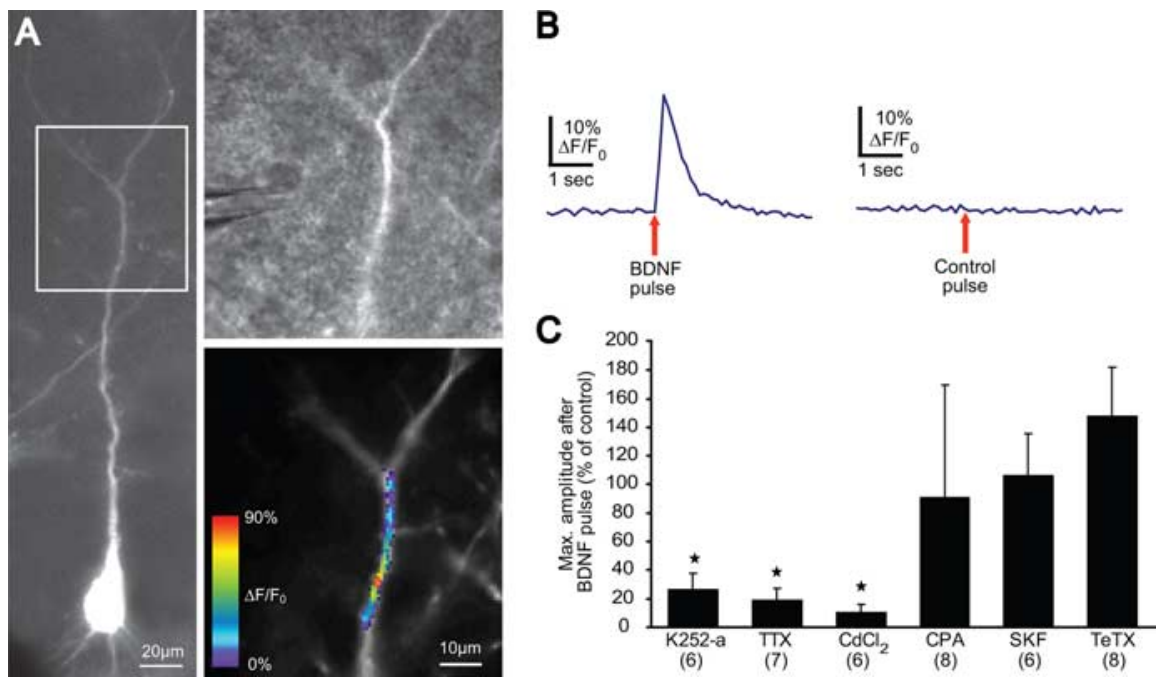

Figure 3. Dendritic calcium transients can be elicited by focal BDNF application and are dependent on activation of TrkB receptors as well as voltage-gated sodium and calcium channels. $\boldsymbol{A}$, An electroporated CA3 neuron with the BDNF application pipette, placed $\sim 20 \mu \mathrm{m}$ from the dendrite (top right), responds to a single $40 \mathrm{~ms}$ BDNF pulse with a strong local increase in $\left[\mathrm{Ca}^{2+}\right]_{\mathrm{i}} \cdot \boldsymbol{B}$, Left trace shows an average of calcium responses to six BDNF pulses applied at the indicated dendritic region in $\boldsymbol{A}$. Right trace shows averaged responses to six pulses of a control solution (heat-inactivated BDNF) in another neuron. C, Pharmacology of BDNF-evoked calcium transients: amplitudes of BDNF-evoked calcium transients are shown as percentage of baseline. Bath application of $\mathrm{K} 252-\mathrm{a}$, $\mathrm{TTX}$, and $\mathrm{CdCl}_{2}$ reduced the amplitude of the calcium transients evoked by local BDNF application significantly $\left({ }^{*} p<0.05\right)$. In contrast, no reduction in the amplitude of the BDNF-induced calcium transients was observed during bath application of CPA, SKF 96365 hydrochloride, or in TeTX incubated slices.

elicited only the immediate calcium response (Fig. $2 E, G$ ), demonstrating that the increase in frequency that we observed in control slices after BDNF application was a consequence of a BDNF-induced modulation of network activity. Fast application of control solution (PBS) caused neither an acute rise in the calcium concentration nor an increase in the frequency of calcium transients (data not shown). Together, these observations show that exogenous BDNF has two effects on CA3 neurons: it (1) directly triggers a transient rise in $\left[\mathrm{Ca}^{2+}\right]_{\mathrm{i}}$ and (2) causes an increase in network activity, probably by modulating synaptic function (Shen et al., 2006; Tyler et al., 2006; Walz et al., 2006).

\section{Focal BDNF application triggers fast and local calcium transients}

To further characterize calcium transients evoked directly by BDNF, we tested its specific action on dendrites of neurons by focally applying BDNF via a micropipette using a Picospritzer. We placed a pipette filled with BDNF $10-20 \mu \mathrm{m}$ away from the dendrite (Fig. 3A) and applied single BDNF pulses ( $40 \mathrm{~ms}, 200$ $\mathrm{ng} / \mathrm{ml}$ ) to the dendrite of neurons exhibiting spontaneous calcium activity. In $\sim 60 \%$ of those neurons, single BDNF pulses elicited dendritic calcium rises (Fig. $3 A$ ). We recorded twice for 1 min at $10 \mathrm{~Hz}$ with an interval of 3 min between acquisitions. During these two recordings, we applied a total of six BDNF pulses to the dendrite. The calcium responses to the six BDNF pulses were averaged for each cell as shown in the left trace in Figure $3 B$. We could repetitively evoke fast (rise time $<100 \mathrm{~ms}$ ) and spatially restricted calcium transients. Only cells that responded to at least two of the six BDNF pulses with a calcium rise and that showed an average response $>5 \% \Delta F / F_{0}$ were analyzed $(n=48)$. We performed control experiments to verify that the observed calcium response was specific for the BDNF application: neither PBS plus $0.1 \%$ BSA alone $(n=5)$ nor heatinactivated $\operatorname{BDNF}(n=10)$ elicited calcium transients in the dendrite (Fig. 3B, right trace). Moreover, focal PBS plus $0.1 \%$
BSA application did not evoke calcium transients in those dendrites that responded to BDNF pulses before and after PBS application ( $n=3$; data not shown).

Next, we determined the mechanisms of fast BDNF-TrkB-mediated calcium signaling by pharmacologically characterizing exogenously BDNF-evoked calcium transients. We measured the maximal amplitude of the calcium response within $1 \mathrm{~s}$ after the BDNF pulse before, during, and after bath applying drugs. K252-a (200 $\mathrm{nM})$ as well as TTX $(1 \mu \mathrm{M})$ and $\mathrm{CdCl}_{2}(5$ $\mu \mathrm{M})$, which block TrkB receptors, voltagegated sodium and calcium channels, respectively, reduced the maximal amplitude of the calcium response after the BDNF pulse significantly (Fig. 3C). In contrast, neither CPA $(20 \mu \mathrm{M})$, an agent that interferes with calcium release from internal stores, nor SKF 96365 hydrochloride $(3 \mu \mathrm{M})$, an unspecific inhibitor of store-operated calcium entry and transient receptor potential channels (Merritt et al., 1990; Li et al., 1999), nor TeTX (20 $\mathrm{nM})$, which blocks presynaptic transmitter release, reduced the amplitude of the calcium response after the BDNF pulse. Together, these pharmacological experiments indicate that activation of the TrkB receptor and voltage-gated sodium as well as calcium channels are required for BDNF-evoked calcium transients.

\section{BDNF-mediated local calcium transients occur at synaptic sites}

The observation that spontaneous BDNF-dependent calcium transients were highly localized along the dendrite (Fig. 1) led us to ask whether these transients occur at specific sites, possibly synapses. Using viral infection (Semliki forest virus) (Ehrengruber et al., 1999), we expressed the postsynaptic marker PSD-95: CFP (Graf et al., 2004) in hippocampal neurons. PSD-95:CFP labeling was apparent in the soma and as puncta along the dendrites. We identified presynaptic counterparts of the dendritic PSD-95:CFP puncta with immunohistochemical staining for synapsin. High-magnification confocal reconstruction in three dimensions allowed us to visualize synaptic structures by colocalization analysis. Seventy-four percent of 122 putative PDS-95: CFP puncta were opposed by at least one positive synapsin punctum, whereas $26 \%$ did not show a clear colocalization (Fig. $4 A$ ). The specificity of colocalization was tested using the reverse order of the synapsin stack rotated by $180^{\circ}$. Here, significantly fewer of the PSD-95 clusters colocalized with presynaptic synapsin puncta ( $16 \%$ colocalization, $84 \%$ non-colocalization; $n=8$ neurons; $p<0.05)$. This indicates that the majority of the PSD-95:CFP puncta are indeed postsynaptic sites.

After electroporation of the calcium indicator into PSD-95: CFP-expressing cells, we were able to visualize postsynaptic sites along the dendrite and spontaneous calcium transients simultaneously. Cellular morphology and the frequency of spontaneously occurring global and local calcium transients in these transfected neurons were similar to uninfected neurons. We frequently observed spontaneous local calcium transients at PSD-95:CFP-expressing sites along the dendrite (Fig. 4B). We 
identified the positions of putative synapses and local calcium transients along the dendrites and calculated the distance between the center of the nearest putative synaptic site and the center of each calcium transient. Almost half of the local calcium rises occurred within a distance of $2 \mu \mathrm{m}$ of a putative synapse (41 of 99 from 8 neurons) (Fig. $4 C$ ), which is consistent with previous observations (Lohmann et al., 2005). The mean distance between visualized PSD-95:CFP sites was $17.3 \pm 1.9$ $\mu \mathrm{m}$, which is in good agreement with numbers that can be deduced from electron microscopy studies of CA1 tissue in the hippocampus (Steward and Falk, 1991). To test whether BDNF triggers local calcium transients specifically at PSD-95 sites, we again used functionblocking antibodies against BDNF to interfere with the BDNF-TrkB signaling pathway. We observed that, during BDNF antibody bath application, the frequency of local calcium transients that occurred within a distance of $<2 \mu \mathrm{m}$ from a putative synaptic site was significantly and reversibly reduced to $65.9 \pm 16.8 \%(n=51)$ (Fig. $4 D$, black bars) compared with baseline. At those synapses that exhibited calcium activity during baseline recordings (21 of 51), calcium transients were completely blocked in most cases (17) or were reduced (two) or remained stable (two) during $\alpha \mathrm{BDNF}$ application. We calculated that intrinsic BDNF signaling occurred at excitatory synapses at a mean rate of 0.22 transients per minute and synapse. In contrast to the reduced frequency of calcium transients at synapses, we did not find a change in the frequency of local transients that occurred $>2 \mu \mathrm{m}$ away from the nearest synapse during BDNF antibody application (Fig. $4 D$, gray bars). Furthermore, there was no significant change in the frequency of global calcium rises during BDNF antibody application (1.44 \pm 0.51 global transients per minute) compared with baseline conditions (1.94 \pm 0.82 global transients per minute; data not shown). Together with the experiments described above, this finding indicates that endogenous BDNF directly triggers local calcium transients but not global calcium rises and that these BDNF-TrkB-dependent local calcium transients occur specifically at synaptic sites.

\section{Local calcium transients elicited at synaptic sites by focal BDNF delivery}

Focal BDNF application demonstrated a dendritic mechanism of the BDNF-evoked calcium responses (Fig. 3C). Additionally, we showed that spontaneous local calcium transients occur at PSD95:CFP sites along the dendrite. Therefore, we tested whether dendrites respond to exogenous BDNF only at postsynaptic sites.
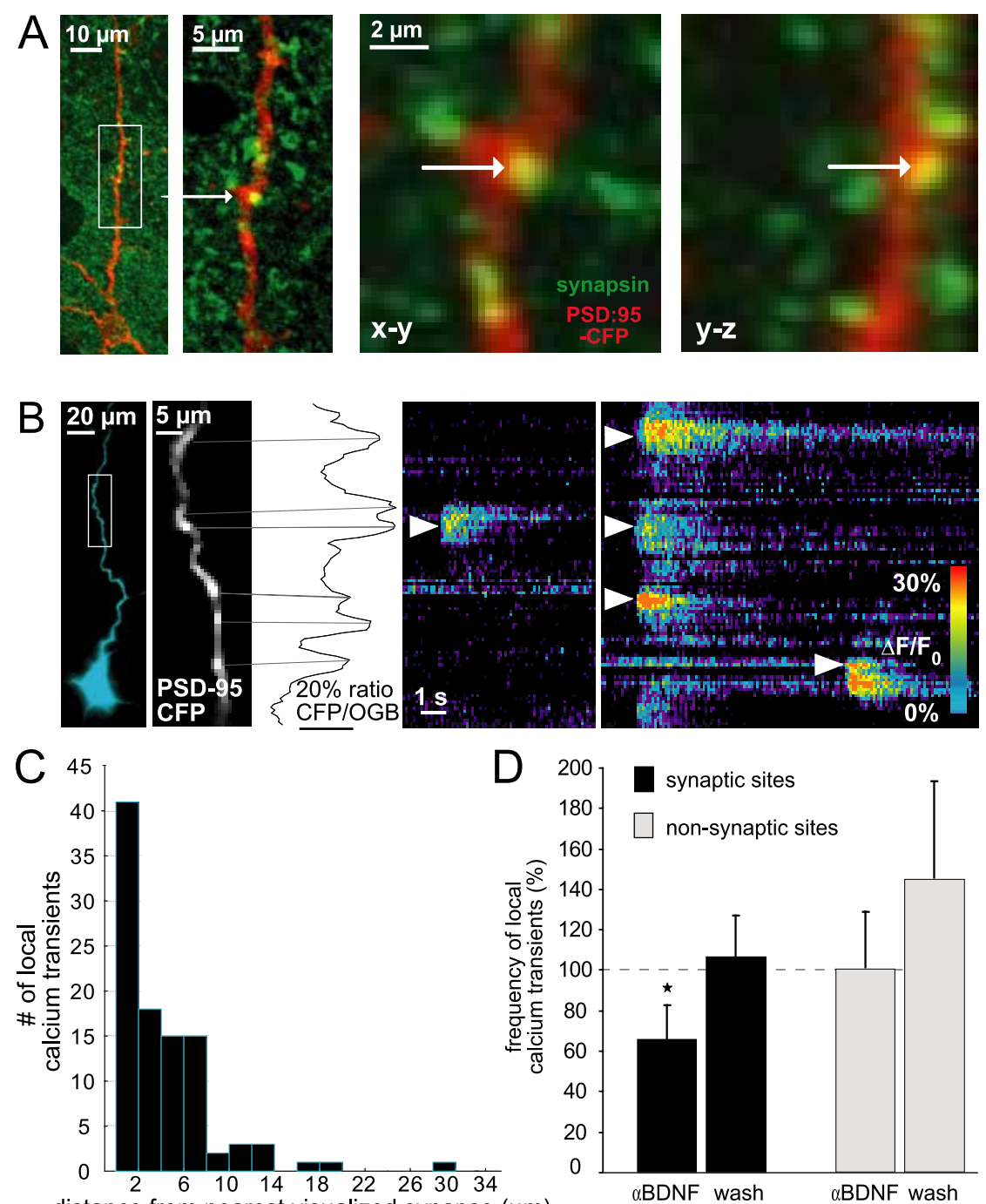

Figure 4. BDNF-mediated local calcium transients occur preferentially at synaptic sites. $\boldsymbol{A}$, Neuron expressing PSD-95:CFP (shown in red) by virus-mediated gene transfer after immunolabeling with anti-synapsin antibodies and high-resolution confocal reconstruction (left, maximum intensity projection of the neuron in red, single plane of synapsin staining in green). Arrow indicates a synapse as PSD-95:CFP and synapsin overlap. High-magnification $(x-y)$ and orthogonal $(y-z)$ views of the marked synapse (yellow pixels in both views). B, PSD-95:CFP (blue) expressing hippocampal neuron electroporated with the calcium indicator Oregon Green BAPTA-1 (OGB) (green). Enlarged part of the dendrite shows PSD-95:CFP puncta; the puncta are also revealed in a ratio plot of CFP/Oregon Green BAPTA-1 pixel intensity along the dendrite. Line scan of the enlarged part of the dendrite shows spontaneously occurring local calcium transients at four of the PSD-95:CFP sites (right). C, Distribution of spontaneous local calcium transients with respect to putative synaptic sites ( 99 local calcium signals from 8 neurons). Most transients occur at or very close to the nearest synapse (the mean intersynapse distance of $17.3 \pm 1.9 \mu \mathrm{m}$ agrees with numbers observed in CA1 neurons in the first postnatal week) (Steward and Falk, 1991). D, Frequency of spontaneous local calcium transients occurring application of BDNF antibodies. The frequency of local calcium transients at synaptic sites, but not at nonsynaptic sites, is significantly reduced during bath application of BDNF antibodies ( $n=8$ neurons; percentage of baseline; ${ }^{*} p<0.05$ ).

Neurons with spontaneous calcium activity were used for these experiments. The tip of a BDNF-filled glass pipette was placed $10-20 \mu \mathrm{m}$ away from the PSD-95 site of the dendrite (Fig. 5A, middle). A single BDNF pulse evoked a strong and localized calcium transient exactly at the PSD-95:CFP sites (Fig. 5A, right). The respective line scan indicates that the BDNF-mediated calcium rise started precisely at the two PSD-95 puncta on the dendrite and reaches the maximum change in fluorescence there (Fig. 5A, bottom). Another example of a BDNF pulse, followed immediately by a localized calcium rise at a synaptic site, is shown in Figure 5B. Here, BDNF evoked a larger signal at the PSD-95: 

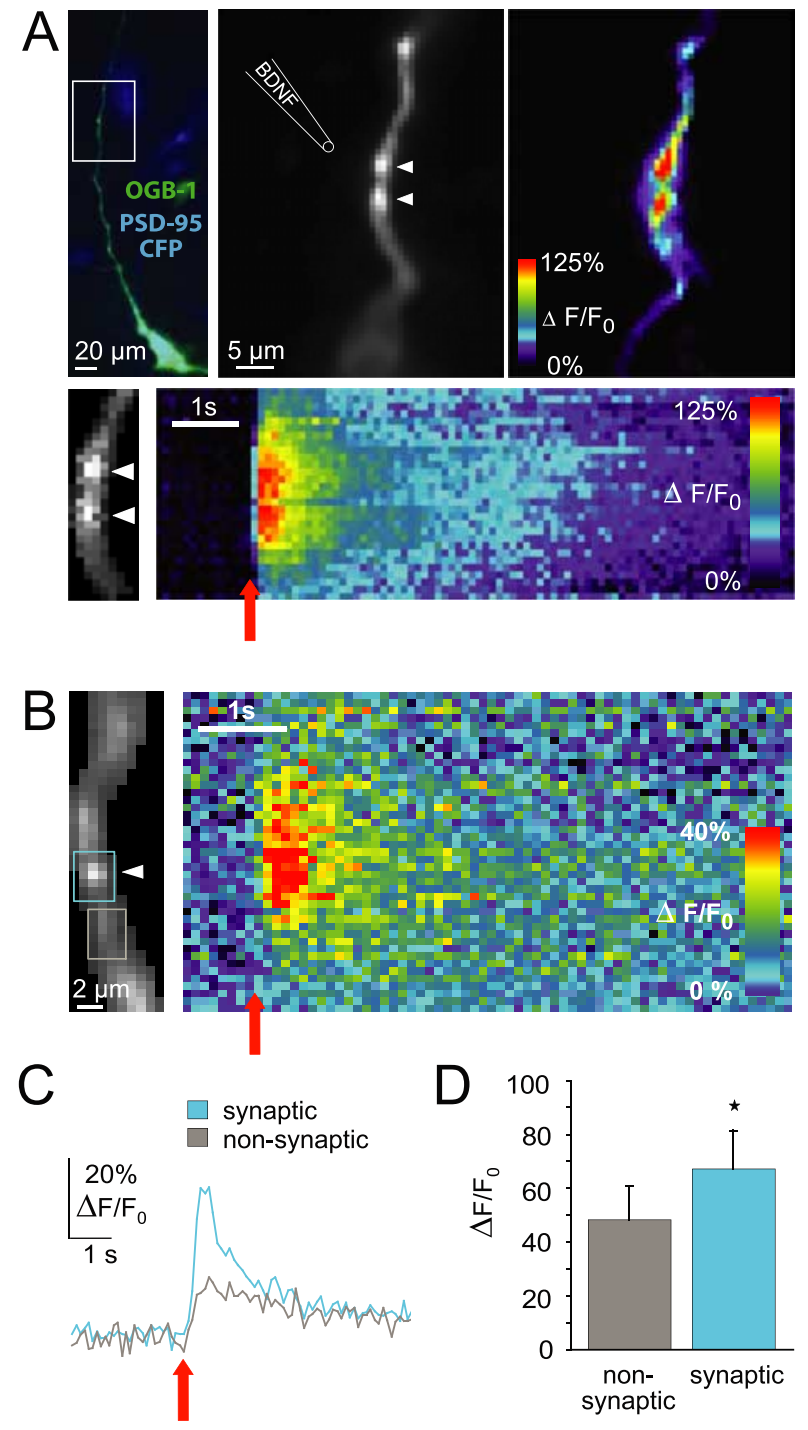

Figure 5. BDNF-evoked local calcium transients can be induced at synaptic sites. $\boldsymbol{A}$, PSD-95: CFP-expressing pyramidal neuron, electroporated with Oregon Green BAPTA-1 (0GB-1) (left); arrowheads mark PSD-95:CFP puncta along the apical dendrite. A BDNF pulse caused a strong calcium rise (represented as $\Delta F / F_{0}$ values) exactly at the sites of two putative synapses (right). Bottom, Line scan reveals that calcium transient induced by the BDNF pulse (red arrow) starts at the two PSD-95:CFP sites and shows exactly there the highest fluorescence increase. $\boldsymbol{B}$, Another example of a BDNF-evoked calcium transient at a PSD-95:CFP site. C, BDNF-evoked calcium transients at a PSD-95:CFP punctum (blue trace) and at an adjacent region (gray trace) indicated in $\boldsymbol{B}$. $\boldsymbol{D}$, Maximal amplitudes of $\Delta F / F_{0}$ within $1 \mathrm{~s}$ after the BDNF pulse at nonsynaptic dendritic sites and at PSD-95:CFP puncta $\left({ }^{*} p<0.05 ; n=9\right)$.

CFP punctum (blue region) than at the adjacent nonsynaptic dendrite (Fig. $5 C$, gray region). On average, the amplitude of the $\Delta F / F_{0}$ signal after the BDNF pulse was significantly higher at the PSD-95:CFP puncta than at the adjacent nonsynaptic dendritic sites (Fig. 5D). These findings show that focal, pulse-like delivery of BDNF evokes localized calcium rises restricted to synaptic sites.

\section{Discussion}

We used calcium imaging to investigate directly intrinsic BDNF signaling in developing hippocampal neurons during synapse formation. Our results provide strong evidence that intrinsic BDNF signaling is highly specific in space and time; endogenous BDNF induces fast calcium transients at synapses in developing dendrites. Furthermore, by quantifying the characteristics of BDNF-triggered calcium transients, we determined constraints for the regulation of developmental processes by fast BDNF signaling. The temporal $(<1 \mathrm{~s})$ and spatial $(<10 \mu \mathrm{m})$ characteristics of intrinsic BDNF signaling as well as its relative abundance ( $>10 / \mathrm{h} /$ synapse) render BDNF an ideal signaling molecule for establishing specific synaptic connectivity and functional neuronal networks.

\section{Intrinsic BDNF signaling is fast}

Blocking intrinsic BDNF signaling in developing hippocampal slices reduced the frequency of spontaneous fast and locally restricted calcium transients. Conversely, focal application of exogenous BDNF elicited fast local calcium transients. Together, these results show that $\mathrm{BDNF}$ is an important mediator of fast local calcium transients in developing dendrites.

What makes BDNF signaling so fast? We investigated the mechanism of BDNF-triggered dendritic calcium transients in developing dendrites of hippocampal neurons and found that they depend on the activation of TrkB and fast opening ion channels, such as voltage-gated sodium and calcium channels. They are not dependent on release of calcium from internal stores. The tetanus toxin experiments exclude the possibility that BDNF may stimulate presynaptic transmitter release, which could in turn induce dendritic calcium transients. Together, our results show that BDNF directly triggers dendritic calcium transients via TrkB and voltage-gated currents. Such a mechanism is reminiscent of the effects of exogenously applied BDNF, which have been described previously. BDNF can cause depolarizations in cultured neurons within a few milliseconds (Kafitz et al., 1999) that require the activation of voltage-dependent sodium channels (Blum et al., 2002) as well as fast calcium transients through the opening of voltage-gated calcium channels (Berninger et al., 1993; Kovalchuk et al., 2002). Our results extend these findings by demonstrating that developing hippocampal neurons intrinsically generate fast BDNF signaling. The onset kinetics of BDNFevoked calcium transients are faster than $100 \mathrm{~ms}$, the temporal resolution of our recordings, suggesting that they are in the range of several tens of milliseconds or less, which is within the temporal requirements for spike-timing-dependent plasticity in the mature nervous system (Dan and Poo, 2004). There is evidence, however, for a reduced requirement for temporal precision in activity-dependent plasticity during development (0.1-1 s) (Lee et al., 2002; Kasyanov et al., 2004). Therefore, the temporal characteristics of BDNF induced calcium transients (rise time $<100$ $\mathrm{ms}$, duration $\sim 700 \mathrm{~ms}$ ) accurately reflect the temporal requirements for activity-dependent synaptic plasticity during development. Furthermore, we show that this phenomenon occurs under natural conditions frequently enough to play a pivotal role in synapse development.

In principle, two scenarios could explain fast intrinsic BDNF signaling: (1) rapid release of BDNF at specific sites, most likely at synapses (see below), triggers a direct response in the dendrite; (2) tonic BDNF release establishes a diffuse distribution of BDNF throughout the tissue and stochastic activation of $\mathrm{TrkB}$ receptors generates fast dendritic calcium transients. Our experiments support the first scenario, because fast and localized application of BDNF triggers immediate calcium rises (Figs. $2 G, 3,5$ ). In contrast, our data are not consistent with the second scenario, because we find that the persistent and diffuse presence of BDNF is not an appropriate stimulus for the direct activation of fast calcium rises (Fig. 2C,E). Desensitization of TrkB receptors might prevent a sustained response during long-term BDNF exposure. 
From these findings, we infer that the induction of fast calcium transients requires the rapid release of BDNF in close vicinity to the dendrite. In addition, these results support the idea that both endogenous BDNF release and dendritic response are very precisely regulated in time.

\section{Intrinsic BDNF signaling is localized to synapses}

We observed that BDNF-evoked calcium transients are localized to specific dendritic sites. Interestingly, simultaneous imaging of putative postsynaptic structures, labeled with PSD-95:CFP, and local calcium transients revealed that BDNF-triggered transients occur primarily or exclusively at synapses.

One potential caveat of virally overexpressing synaptic PSD-95 is that it may affect health of infected neurons. Additionally, it might change signaling as it increases the number of synaptic AMPA receptors as shown previously (Schnell et al., 2002). The increase in synaptic AMPA receptors will most likely not influence the localization of TrkB because no direct interaction between TrkB and AMPA receptors or PSD-95 has been shown. Furthermore, we observed no difference in the structure or function between transfected and wild-type cells, indicating that overexpression did not affect viability or signaling. (1) The cellular morphology of transfected pyramidal neurons was not different from nontransfected neurons. (2) The frequencies of spontaneously occurring global and local calcium transients were similar in normal and overexpressing neurons. (3) The proportion of local calcium transients occurring at synapses was similar to that found in a previous study using postfixation immunohistochemistry instead of PSD-95:CFP for labeling synapses (Lohmann et al., 2005). (4) The proportion of local calcium transients that were blocked by interfering with BDNF signaling was similar in normal and transfected cells. Together with our finding that most PSD-95:CFP puncta were opposed by synapsin-positive structures, these observations confirm that virally overexpressed PSD95:CFP is an appropriate label for identifying postsynaptic signaling.

We found BDNF-induced calcium transients at synaptic sites during intrinsic BDNF signaling and after focal delivery of BDNF. The second observation implicates that dendrites are particularly sensitive to BDNF at synapses. The most straightforward explanation would be the specific presence of TrkB at the synapse. This fits well with an immunoelectron microscopical study that reported TrkB immunoreactivity predominantly at synapses within the dendrites of hippocampal pyramidal neurons (Drake et al., 1999). TrkB, which resides at nonsynaptic sites along dendrites, is mostly confined to intracellular membranes (Drake et al., 1999), suggesting that functional receptors are enriched at synaptic sites (Gomes et al., 2006). This predominantly synaptic distribution of TrkB likely underlies the local specificity of BDNF-evoked calcium transients observed here.

Although our results implicate that endogenous BDNF is released near the synapse and directly triggers calcium transients at the postsynapse, it is not clear whether BDNF originates from the presynapse or postsynapse. Evidence for both modes of release has been obtained (Hartmann et al., 2001; Kohara et al., 2001; Lessmann et al., 2003; Gartner et al., 2006). Independently of the site of release, it has been shown previously that BDNF regulates neuronal development on a local as well as on a global level. The here identified intrinsic form of fast BDNF signaling occurs at synaptic sites and extends only a few micrometers along the dendrite, much less than the average distance between excitatory synapses at these early stages of synapse development. This observation implicates intrinsic BDNF signaling in regulating the development of single synapses, possibly by mediating synaptic maturation (Fig. 3) (Rutherford et al., 1998; Itami et al., 2003; Walz et al., 2006). In addition, because TrkB activates diverse intracellular signaling pathways, the intriguing possibility arises that BDNF-TrkB signaling activates parallel pathways to regulate local and global as well as rapid and slower aspects of neuronal development differentially.

Our results also indicate that BDNF is one of several factors, which contribute to local dendritic calcium activity, because only a fraction of local dendritic calcium transients is blocked by interfering with intrinsic BDNF signaling. We conclude that developing neurons are able to respond to a number of different extracellular cues during development and that they integrate those by generating very specific patterns of local and global calcium transients. Such multifactorial calcium transients may regulate structural development and synapse formation and eventually help establish the complex neuronal networks of the brain.

\section{References}

Balkowiec A, Katz DM (2002) Cellular mechanisms regulating activitydependent release of native brain-derived neurotrophic factor from hippocampal neurons. J Neurosci 22:10399-10407.

Berninger B, Garcia DE, Inagaki N, Hahnel C, Lindholm D (1993) BDNF and NT-3 induce intracellular $\mathrm{Ca}^{2+}$ elevation in hippocampal neurones. NeuroReport 4:1303-1306.

Bibel M, Barde YA (2000) Neurotrophins: key regulators of cell fate and cell shape in the vertebrate nervous system. Genes Dev 14:2919-2937.

Blum R, Kafitz KW, Konnerth A (2002) Neurotrophin-evoked depolarization requires the sodium channel $\mathrm{Na}(\mathrm{V}) 1.9$. Nature 419:687-693.

Dailey ME, Smith SJ (1996) The dynamics of dendritic structure in developing hippocampal slices. J Neurosci 16:2983-2994.

Dan Y, Poo MM (2004) Spike timing-dependent plasticity of neural circuits. Neuron 44:23-30.

DiCiommo DP, Bremner R (1998) Rapid, high level protein production using DNA-based semliki forest virus vectors. J Biol Chem 273:18060-18066.

Drake CT, Milner TA, Patterson SL (1999) Ultrastructural localization of full-length trkB immunoreactivity in rat hippocampus suggests multiple roles in modulating activity-dependent synaptic plasticity. J Neurosci 19:8009-8026.

Ehrengruber MU, Lundstrom K, Schweitzer C, Heuss C, Schlesinger S, Gahwiler BH (1999) Recombinant Semliki Forest virus and Sindbis virus efficiently infect neurons in hippocampal slice cultures. Proc Natl Acad Sci USA 96:7041-7046.

Fiala JC, Feinberg M, Popov V, Harris KM (1998) Synaptogenesis via dendritic filopodia in developing hippocampal area CA1. J Neurosci 18:8900-8911.

Garaschuk O, Hanse E, Konnerth A (1998) Developmental profile and synaptic origin of early network oscillations in the CA1 region of rat neonatal hippocampus. J Physiol (Lond) 507:219-236.

Gartner A, Staiger V (2002) Neurotrophin secretion from hippocampal neurons evoked by long-term-potentiation-inducing electrical stimulation patterns. Proc Natl Acad Sci USA 99:6386-6391.

Gartner A, Polnau DG, Staiger V, Sciarretta C, Minichiello L, Thoenen H, Bonhoeffer T, Korte M (2006) Hippocampal long-term potentiation is supported by presynaptic and postsynaptic tyrosine receptor kinase B-mediated phospholipase C $\gamma$ signaling. J Neurosci 26:3496-3504.

Gomes RA, Hampton C, El Sabeawy F, Sabo SL, McAllister AK (2006) The dynamic distribution of TrkB receptors before, during, and after synapse formation between cortical neurons. J Neurosci 26:11487-11500.

Gorski JA, Zeiler SR, Tamowski S, Jones KR (2003) Brain-derived neurotrophic factor is required for the maintenance of cortical dendrites. J Neurosci 23:6856-6865.

Graf ER, Zhang X, Jin SX, Linhoff MW, Craig AM (2004) Neurexins induce differentiation of GABA and glutamate postsynaptic specializations via neuroligins. Cell 119:1013-1026.

Haas K, Sin WC, Javaherian A, Li Z, Cline HT (2001) Single-cell electroporation for gene transfer in vivo. Neuron 29:583-591.

Hartmann M, Heumann R, Lessmann V (2001) Synaptic secretion of BDNF 
after high-frequency stimulation of glutamatergic synapses. EMBO J 20:5887-5897.

Horch HW, Katz LC (2002) BDNF release from single cells elicits local dendritic growth in nearby neurons. Nat Neurosci 5:1177-1184.

Hu B, Nikolakopoulou AM, Cohen-Cory S (2005) BDNF stabilizes synapses and maintains the structural complexity of optic axons in vivo. Development 132:4285-4298.

Huang EJ, Reichardt LF (2001) Neurotrophins: roles in neuronal development and function. Annu Rev Neurosci 24:677-736.

Itami C, Kimura F, Kohno T, Matsuoka M, Ichikawa M, Tsumoto T, Nakamura S (2003) Brain-derived neurotrophic factor-dependent unmasking of "silent" synapses in the developing mouse barrel cortex. Proc Natl Acad Sci USA 100:13069-13074.

Kafitz KW, Rose CR, Thoenen H, Konnerth A (1999) Neurotrophin-evoked rapid excitation through TrkB receptors. Nature 401:918-921.

Kang H, Schuman EM (1995) Long-lasting neurotrophin-induced enhancement of synaptic transmission in the adult hippocampus. Science 267:1658-1662.

Kang H, Schuman EM (2000) Intracellular $\mathrm{Ca}(2+)$ signaling is required for neurotrophin-induced potentiation in the adult rat hippocampus. Neurosci Lett 282:141-144.

Kasyanov AM, Safiulina VF, Voronin LL, Cherubini E (2004) GABAmediated giant depolarizing potentials as coincidence detectors for enhancing synaptic efficacy in the developing hippocampus. Proc Natl Acad Sci USA 101:3967-3972.

Kohara K, Kitamura A, Morishima M, Tsumoto T (2001) Activitydependent transfer of brain-derived neurotrophic factor to postsynaptic neurons. Science 291:2419-2423.

Kolbeck R, Bartke I, Eberle W, Barde YA (1999) Brain-derived neurotrophic factor levels in the nervous system of wild-type and neurotrophin gene mutant mice. J Neurochem 72:1930-1938.

Korte M, Carroll P, Wolf E, Brem G, Thoenen H, Bonhoeffer T (1995) Hippocampal long-term potentiation is impaired in mice lacking brainderived neurotrophic factor. Proc Natl Acad Sci USA 92:8856-8860.

Korte M, Staiger V, Griesbeck O, Thoenen H, Bonhoeffer T (1996) The involvement of brain-derived neurotrophic factor in hippocampal longterm potentiation revealed by gene targeting experiments. J Physiol (Paris) 90:157-164.

Kossel AH, Cambridge SB, Wagner U, Bonhoeffer T (2001) A caged Ab reveals an immediate/instructive effect of BDNF during hippocampal synaptic potentiation. Proc Natl Acad Sci USA 98:14702-14707.

Kovalchuk Y, Hanse E, Kafitz KW, Konnerth A (2002) Postsynaptic induction of BDNF-mediated long-term potentiation. Science 295:1729-1734.

Lee CW, Eglen SJ, Wong RO (2002) Segregation of ON and OFF retinogeniculate connectivity directed by patterned spontaneous activity. J Neurophysiol 88:2311-2321.

Lessmann V, Gottmann K, Malcangio M (2003) Neurotrophin secretion: current facts and future prospects. Prog Neurobiol 69:341-374.

Levine ES, Dreyfus CF, Black IB, Plummer MR (1995) Brain-derived neurotrophic factor rapidly enhances synaptic transmission in hippocampal neurons via postsynaptic tyrosine kinase receptors. Proc Natl Acad Sci USA 92:8074-8077.

Levine ES, Crozier RA, Black IB, Plummer MR (1998) Brain-derived neurotrophic factor modulates hippocampal synaptic transmission by increasing $N$-methyl-D-aspartic acid receptor activity. Proc Natl Acad Sci USA 95:10235-10239.
Li HS, Xu XZ, Montell C (1999) Activation of a TRPC3-dependent cation current through the neurotrophin BDNF. Neuron 24:261-273.

Lohmann C, Finski A, Bonhoeffer T (2005) Local calcium transients regulate the spontaneous motility of dendritic filopodia. Nat Neurosci 8:305-312.

Lohof AM, Ip NY, Poo MM (1993) Potentiation of developing neuromuscular synapses by the neurotrophins NT-3 and BDNF. Nature 363:350-353.

Marsh HN, Palfrey HC (1996) Neurotrophin-3 and brain-derived neurotrophic factor activate multiple signal transduction events but are not survival factors for hippocampal pyramidal neurons. J Neurochem 67:952-963.

McAllister AK, Lo DC, Katz LC (1995) Neurotrophins regulate dendritic growth in developing visual cortex. Neuron 15:791-803.

McAllister AK, Katz LC, Lo DC (1999) Neurotrophins and synaptic plasticity. Annu Rev Neurosci 22:295-318.

Merritt JE, Armstrong WP, Benham CD, Hallam TJ, Jacob R, Jaxa-Chamiec A, Leigh BK, McCarthy SA, Moores KE, Rink TJ (1990) SKF 96365, a novel inhibitor of receptor-mediated calcium entry. Biochem J 271:515-522.

Patterson SL, Abel T, Deuel TA, Martin KC, Rose JC, Kandel ER (1996) Recombinant BDNF rescues deficits in basal synaptic transmission and hippocampal LTP in BDNF knockout mice. Neuron 16:1137-1145.

Poo MM (2001) Neurotrophins as synaptic modulators. Nat Rev Neurosci 2:24-32.

Rathenberg J, Nevian T, Witzemann V (2003) High-efficiency transfection of individual neurons using modified electrophysiology techniques. J Neurosci Methods 126:91-98.

Rutherford LC, Nelson SB, Turrigiano GG (1998) BDNF has opposite effects on the quantal amplitude of pyramidal neuron and interneuron excitatory synapses. Neuron 21:521-530.

Sakai N, Yamada M, Numakawa T, Ogura A, Hatanaka H (1997) BDNF potentiates spontaneous $\mathrm{Ca}^{2+}$ oscillations in cultured hippocampal neurons. Brain Res 778:318-328.

Schnell E, Sizemore M, Karimzadegan S, Chen L, Bredt DS, Nicoll RA (2002) Direct interactions between PSD-95 and stargazin control synaptic AMPA receptor number. Proc Natl Acad Sci USA 99:13902-13907.

Shen W, Wu B, Zhang Z, Dou Y, Rao ZR, Chen YR, Duan S (2006) Activityinduced rapid synaptic maturation mediated by presynaptic cdc 42 signaling. Neuron 50:401-414.

Steward O, Falk PM (1991) Selective localization of polyribosomes beneath developing synapses: a quantitative analysis of the relationships between polyribosomes and developing synapses in the hippocampus and dentate gyrus. J Comp Neurol 314:545-557.

Stoppini L, Buchs P-A, Muller D (1991) A simple method for organotypic cultures of nervous tissue. J Neurosci Methods 37:173-182.

Stosiek C, Garaschuk O, Holthoff K, Konnerth A (2003) In vivo two-photon calcium imaging of neuronal networks. Proc Natl Acad Sci USA 100:7319-7324.

Tyler WJ, Zhang XL, Hartman K, Winterer J, Muller W, Stanton PK, PozzoMiller L (2006) BDNF increases release probability and the size of a rapidly recycling vesicle pool within rat hippocampal excitatory synapses. J Physiol (Lond) 574:787-803.

Walz C, Jungling K, Lessmann V, Gottmann K (2006) Presynaptic plasticity in an immature neocortical network requires NMDA receptor activation and BDNF release. J Neurophysiol 96:3512-3516. 